\title{
Embryonic and mesenchymal stem cells:An examination of the rapidly narrowing gap between their proven abilities
}

\begin{abstract}
It has become clear that adult mammalian organisms contain populations of adult stem cells, one of which can be called mesenchymal stem cells (MSCs). These cells are undifferentiated, have the ability to self-renew, differentiate into other cell types, and contribute to the maintenance and regeneration of tissue. Over the past decade, multiple studies have demonstrated the ability of MSCs to differentiate into non-mesenchymal lineages, challenging the previously held assumption that only embryonic stem cells (ESCs) were able to differentiate into all three germ layers. Due to their ease of isolation, ease of expansion in vitro, and wide differentiation potential, MSCs have become an attractive cell source for tissue engineering applications. The use of mesenchymal stem cells represents an important opportunity for the development of new therapies using stem cell technologies.
\end{abstract}

Volume 4 Issue I - 2018

\author{
Amanda Baracho Trindade Hill, Joaquim \\ Mansano Garcia \\ Sao Paulo State University, Brazil
}

Correspondence: Amanda Baracho Trindade Hill, Sao Paulo State University - FCAV, Jaboticabal-SP, Brazil,Tel +5 51699712 0331,Email amanda.baracho@hotmail.com

Received: March 06, 2018 | Published: March 12, 2018

\section{Short Communication}

Stem cell therapy and biology have displayed increasingly concrete potential resolutions to a diversity of obstacles in the treatment of chronic and degenerative diseases over the past decade. Encouraging results continue to be generated, further increasing the amount of interest in the field and the number of studies performed, giving hope to patients with, until now, incurable chronic and degenerative diseases with few new ideas on the horizon.

Stem cells are defined by their ability to self-renew and to differentiate. These cells are responsible for the development of the organism where, in the early embryo, the stem cells in the inner cell mass differentiate into all cell types that form the body. While, the trophectoderm cells differentiate into extra embryonic lineage. ${ }^{1}$ In an adult body, stem cells are known as mesenchymal stem cells (MSCs) and regulate tissue turnover. Those cells have been described as having a restricted potential for differentiation when compared to embryonic stem cells (ESCs) that, as previously mentioned, can differentiate into all cell types in an organism. ${ }^{1}$ Mesenchymal stem cells have received a lot of attention lately, specifically due to the facts that they are easy to isolate, have tropic and immunosuppressive effects, and have the potential for triploblastic differentiation under the right conditions. ${ }^{2}$ On the contrary, the use of ESCs involves ethical problems due to the fact that their harvest requires the destruction of an embryo; and they also present a technical barrier in that any treatments using ESCs will not be autologous. According to Gazit et al. ${ }^{3}$ MSCs are the subject of an ongoing public discussion involving concerns and challenges regarding the veracity of the definition of their comparatively limited differentiability, the central question being the potential of differentiation into cells that are not of mesenchymal nature. Over the past decade, it has been questioned whether the differences that previously seemed to exist between embryonic and mesenchymal stem cells do exist at all. Many studies have shown evidence that MSCs can differentiate into all germ layers, further increasing research and clinical interest regarding $\mathrm{MSCs}^{4}$.

Many researchers have focused their efforts on MSCs, attempting to elucidate better ways to grow a large amount of adult stem cells in laboratories and to induce differentiation of specific cell types; targeting specific treatments of diseases or repair of tissue injuries. ${ }^{5}$ Our group showed that canine MSCs were able to differentiate into endodermal, ectodermal, mesodermal and even primordial germ cell precursors in vitro. ${ }^{6}$ Many other studies have also shown the potential of MSCs to differentiate into all non mesodermal lineages with the notable and clinically advantageous exception of teratomas, further increasing clinical interest. ${ }^{2}$

Finding reliable sources of cells with wide differentiation capacity in adult organisms would provide a promising tool for regenerative medicine, specifically in facilitating autologous treatment.

\section{Acknowledgements}

None

\section{Conflict of interest}

Author declares that there is no conflict of interest.

\section{References}

1. Gattegno-Ho D, Argyle SA, Argyle DJ. Stem cells and veterinary medicine: Tools to understand diseases and enable tissue regeneration and drug discovery. The Veterinary Journal. 2012;191(1):19-27.

2. Kuroda Y, Dezawa M. Mesenchymal stem cells and their subpopulation, pluripotent muse cells, in basic research and regenerative medicine. Anat Rec (Hoboken). 2014;297(1):98-110.

3. Gazit Z, Pelled G, Sheyn D, et al. Principles of Regenerative Medicine. Mesenchymal Stem Cells. 2011;17:285-304.

4. Zipori D. The nature of stem cells: state rather than entity. Nat Rev Genet 2004;5(11):873-878.

5. Sharma N, Jeong DK. Stem cell research: a novel boulevard towards improved bovine mastitis management. International journal of biological sciences. 2013;9(8):818-829.

6. Hill ABT, Therrien J, Garcia JM, et al. Mesenchymal-like stem cells in canine ovary show high differentiation potential. Cell Prolif. 2017;50(6). 